\title{
Discrimination of Direction in Fast Frequency-Modulated Tones by Rats
}

\author{
Bernhard H. Gaese, ${ }^{1,3}$ Isabella King, ${ }^{1}$ Christian Felsheim, ${ }^{2}$ Joachim Ostwald, ${ }^{2}$ \\ AND WOLFGER VON DER BEHRENS ${ }^{3}$ \\ ${ }^{1}$ Institut für Biologie II, RWTH Aachen, Kopernikusstr. 16, D-52074 Aachen, Germany \\ ${ }^{2}$ Lehrstuhl Tierphysiologie, Universität Tübingen, Auf der Morgenstelle 28, D-72076 Tübingen, Germany \\ ${ }^{3}$ Zoologisches Institut, J.W. Goethe Universität, Siesmayerstr. 70, D-60323 Frankfurt a.M., Germany
}

Received: 11 July 2005; Accepted: 16 November 2005; Online publication: 13 January 2006

\begin{abstract}
Fast frequency modulations (FM) are an essential part of species-specific auditory signals in animals as well as in human speech. Major parameters characterizing non-periodic frequency modulations are the direction of frequency change in the FM sweep (upward/downward) and the sweep speed, i.e., the speed of frequency change. While it is well established that both parameters are represented in the mammalian central auditory pathway, their importance at the perceptual level in animals is unclear. We determined the ability of rats to discriminate between upward and downward modulated FM-tones as a function of sweep speed in a two-alternative-forcedchoice-paradigm. Directional discrimination in logarithmic FM-sweeps was reduced with increasing sweep speed between 20 and 1,000 octaves/s following a psychometric function. Average threshold sweep speed for FM directional discrimination was 96 octaves/s. This upper limit of perceptual FM discrimination fits well the upper limit of preferred sweep speeds in auditory neurons and the upper limit of neuronal direction selectivity in the rat auditory cortex and midbrain, as it is found in the literature. Influences of additional stimulus parameters on FM discrimination were determined using an adaptive testing-procedure for efficient threshold estimation based on a maximum likelihood approach. Directional discrimination improved with extended FM
\end{abstract}

Correspondence to: Bernhard H. Gaese $•$ Zoologisches Institut • J.W. Goethe Universität - Siesmayerstr. 70, D-60323 Frankfurt a.M., Germany. Telephone: +49-69-79824742; fax: +49-69-79824750; email: gaese@zoology.uni-frankfurt.de sweep range between two and five octaves. Discrimination performance declined with increasing lower frequency boundary of FM sweeps, showing an especially strong deterioration when the boundary was raised from 2 to $4 \mathrm{kHz}$. This deterioration corresponds to a frequency-dependent decline in direction selectivity of FM-encoding neurons in the rat auditory cortex, as described in the literature. Taken together, by investigating directional discrimination of FM sweeps in the rat we found characteristics at the perceptual level that can be related to several aspects of FM encoding in the central auditory pathway.

Keywords: FM sweep, direction discrimination, two-alternative-forced choice, auditory-threshold, adaptive-procedure

\section{INTRODUCTION}

Dynamic changes are among the most prominent stimulus features in naturally occurring acoustic signals. In particular, frequency modulations (FMs) are important elements of acoustic signaling in various species such as song birds (Marler 2004), bats (Schnitzler and Kalko 2001), monkeys (Moody et al. 1986; May et al. 1989), and even rats (Sales and Pye 1974; Kaltwasser 1990). In human speech, fast FM transients are particularly crucial for phonetic discrimination. Discriminating, for example, between the consonant-vowel syllables [da] and [ga] depends mainly on the slope of the initial time segment of the third formant, with a descending FM sweep in [da] 
and an ascending FM sweep in [ga] (Liberman and Mattingly 1989).

Due to their importance in acoustic communication, the underlying neural representation of fast FM sweeps has been investigated in great detail in several mammalian species. Populations of neurons with preferences for FM sweep direction and/or specific ranges of sweep speeds were found at the cortical and subcortical level, e.g., in the rat (Møller 1969; Gaese and Ostwald 1995; Felsheim and Ostwald 1996; Ricketts et al. 1998; Zhang et al. 2003), the cat (Whitfield and Evans 1965; Kelly and Whitfield 1971; Mendelson and Cynader 1985; Heil et al. 1992b), or the ferret (Nelken and Versnel 2000). The most interesting pattern found is a systematic representation of preferred sweep direction along the tonotopic gradient in the forebrain (low-frequency neurons preferring upward and high-frequency neurons preferring downward FMs), originally described by Heil in the chick (Heil et al. 1992a) and recently also found in the rat (Zhang et al. 2003). Topographic representations of sweep speed and sweep direction that were independent of tonotopy, on the other hand, were found in the mouse inferior colliculus (Hage and Ehret 2003) and in the cat auditory cortex (Mendelson et al. 1993).

In spite of the numerous physiological studies, psychophysical data on the perception of FM components in these animal models are almost completely lacking. No data exist on the importance of stimulus parameters such as sweep speed or sweep direction for the perceptual discrimination of FM components. Only the fact, that animals have the ability for categorical discrimination of FM stimuli (upward vs. downward) was described for the Mongolian gerbil (Wetzel et al. 1998b) and the rat (Mercado et al. 2005). This ability is impaired by right auditory cortex lesion (Wetzel et al. 1998a).

The psychophysics of FM components and the importance of specific stimulus parameters was so far only investigated in detail in humans. Most of all, strong evidence supports the concept of two separate perceptual processing channels for upward and downward FM sweeps which might be the basis for directional discrimination (Gardner and Wilson 1979). Further analysis was mostly done using FM stimuli with low sweep speeds (slow FM). It was found that discrimination of slow FMs is mainly limited by sweep range, not duration (Madden and Fire 1997). Fast FM sweeps, on the other hand, with high sweep speed have larger sweep ranges (more than 10\% of center frequency, well above frequency difference limen). Such fast FMs were used in this study. Their discrimination is mainly limited by FM sweep duration (Schouten 1985). The question, if there is an advantage in detectability or discriminability for either upward or downward FMs remains controversial (Gardner and Wilson 1979; Cullen and Collins 1982; Dooley and Moore 1988; Madden and Fire 1997). Moore and Sek (1998), found no difference in discriminability in a well-controlled experiment.

As any systematic data on the psychophysics of FM direction discrimination in animals are lacking, the goal of this study was to determine the perceptual basis of FM discrimination in a laboratory animal, where psychophysical data can be related to the well investigated physiological basis. We choose the laboratory rat, as it is well suited to be trained in behavioral tasks. Techniques are available for the rat that might finally enable us to investigate the neuronal basis of auditory perception in experiments that combine behavioral testing and the recording of brain activity in parallel (Gaese and Ostwald 2003).

\section{MATERIALS AND METHODS}

\section{Animals}

Five female Sprague-Dawley rats (supplied by inhouse breeding program, Animal care facilities, RWTH Aachen), weight range 240-260 g (i.e., around 3 months old) at the start of the study, were used. The rats were maintained at a shifted $12 \mathrm{hr}$ light/dark cycle (lights on at 07.00 P.M.) and a restricted diet of standard laboratory chow that kept their weight at about $90 \%$ of free-feeding weight. Water was given ad libitum. Training was performed during the dark phase in sessions of about 50-60 min duration, 5 days a week. The care and use of the animals reported on in this study was according to the European Communities Council Directive of 24 November 1986 (86/609/EEC) and followed German federal regulations. Procedures of animal experimentation were approved by the Regierungspräsidium Köln (Germany).

\section{Apparatus}

The test apparatus was a modified standard rat home cage $(32 \times 27 \times 19 \mathrm{~cm})$ with a front wall that included the following elements: one central hole, where the animal started a trial with a nose poke and where it stayed during stimulus delivery; two peripheral holes, one to each side, where the animal indicated its choice (left, right) for a given discrimination with a nose poke; five headphone speakers (RP-HVT11, Panasonic, 20-22 kHz), three above the holes and two in between, that were used to deliver acoustic stimuli during training; 1 LED above the central hole ("ready-LED") that indicated the possibility to initiate a new trial after the time-out between trials. Small amounts of sucrose solution (10\% 
sucrose in water, initially $50 \mu \mathrm{l}$, during final testing about $5 \mu \mathrm{l}$ per trial) were given as a reward through cannulae that were positioned inside the two peripheral holes. At the front of each hole there was a photoelectric cell which registered breaks in an infrared beam inside the hole.

The experimental cage was placed in a soundattenuating box (custom built). The chamber was completely dark most of the time, except when the "ready-LED" came on that indicated that a new trial could be initiated. The animals were observed during training using an infrared video camera.

\section{Acoustic stimulation}

During final testing, acoustic stimuli were delivered from a central loudspeaker (EAS 10 TH 400A, Technics) located $12 \mathrm{~cm}$ above the animal's head. The frequency-response of this stimulus-generating system was essentially flat $(+/-6 \mathrm{~dB})$ between 1 and $50 \mathrm{kHz}$ and rolled of at $10 \mathrm{~dB} /$ octave beyond that. Measurements of the sound pressure level (SPL; in decibel re $20 \mu \mathrm{Pa}$ ) were obtained using a Bruel \& Kjaer $1 / 4^{\prime \prime}$ condenser microphone with preamplifier 2804 situated at the position of the rat's head. Stimulus intensity was adjusted to $60 \mathrm{~dB}$ SPL. Distortion components were not detectable at least $60 \mathrm{~dB}$ below the peak signal level. Background noise level during testing was around $10 \mathrm{~dB}$ SPL (measured several times).

FM tone stimuli were generated using System II and System 3 components from TDT (TDT, Gainsville, FL). Stimuli were calculated in an RP2 signal processor (sampling rate $100 \mathrm{kHz}$ ), attenuated (PA5) and then delivered to one of five possible loudspeakers using a SS1 signal switcher and a broad-band amplifier (WPA-600 Pro, Conrad Electronic, Germany).

In order to investigate FM perception in rats, logarithmic FM sweeps (i.e., log frequency changes linearly in time) were used in this study. They bear a close resemblance to the logarithmic frequency organization of the basilar membrane (for the rat: Müller 1991) and the central auditory pathway (Friauf 1992), thereby resulting in similar FM sweep parameters across the range of frequencies examined. Most relevant for this study, logarithmic FM sweeps have perceptually 'constant speed': any octave is traversed in the same time as any other octave. Logarithmic FM sweeps were sometimes also called 'exponential FM' (Mendelson et al. 1993; Felsheim and Ostwald 1996).

Logarithmic FM sweeps (base 2) were presented with variable sweep speeds (10 to 1,000 octaves/s) and a frequency content in the range between 1,024 and $32,768 \mathrm{~Hz}$. FM stimuli started and terminated with an appended constant frequency section during rise and decay time (5 ms) in order to prevent any interaction between frequency modulation and the onset or offset of the stimulus. One control experiment tested the influence of this appended constant frequency section on FM perception.

\section{Training regimen}

Animals were trained in a two-alternative-forcedchoice procedure (2AFC) to discriminate between upward and downward FM sweeps. Training was computer-controlled using custom-built software that interacted with the apparatus via a digital I/O-card (DT-340, Data Translation, Marlboro, MA) and controlled delivery of acoustic stimuli from the TDT system. (see above)

The responses of the rats were shaped in several steps. The first training program consisted of trials in which short series of acoustic stimuli were presented from the loudspeakers above the reward sites (random change of sides between trials). The animal turned off the series by collecting a reward from the site below the active loudspeaker. In the second training step, the rat first had to place its nose in the central hole and maintain it there for a brief delay. After that, the stimulus was delivered randomly from one side and the animal was rewarded in case of a correct choice. Premature withdrawal from the central hole (break error) or incorrect choice (fault error) resulted in a "time out" punishment and no food reward. Easy to discriminate FM tones (sweep speed 12 octaves/s) were introduced at the next stage. FM tones with upward sweep direction came from the left, FM tones with downward sweep direction came from the right loudspeaker, thereby associating the two different types of FM stimuli with the two different reward sites. To inhibit the animals from generating a side preference, stimuli were presented according to Gellermann (1933) with, for example, no more than three consecutive trials presenting a stimulus with the same FM direction. In the fourth training step, an increasing percentage of FM tones of different sweep direction were presented from the central loudspeaker. Finally, different FM tones with increasing sweep speeds up to 1,000 octaves/s (and increasing difficulty to differentiate) were presented from the central speaker only.

\section{Behavioral testing procedures and estimate of thresholds}

To start a trial in the testing paradigm, the animal was required to poke its nose into the central hole. After $350 \mathrm{~ms}$ (in some animals a randomized delay between 50 and $250 \mathrm{~ms}$ was used, but no general difference in the performance was obvious), an FM 
tone was presented from a loudspeaker right above the animal. To complete a correct trial the rat withdrew from the central hole and moved to one of the peripheral holes, to the left for upward FM and to the right for downward FM. The animal indicated its choice with a nose poke and received a reward if correct. After that the rat had to stay away from the three holes for $500 \mathrm{~ms}$ before a new trial could be initiated. Break and fault errors resulted now in a timeout of 5,000 ms. The animals were trained until they showed stable performance of discrimination and a break rate below $20 \%$.

In the first part of the study, we used a 2AFC paradigm with the method of constant stimuli to obtain the animals' psychometric function for discriminating FM tones of different sweep speeds (20, 30, 40, $50,60,70,80,90,100,120,200,500,1,000$ octaves $/ \mathrm{s}$ ) according to their sweep direction (up/down). Different sweep speeds were presented in random succession. A psychometric function based on 100 trials per sweep speed and direction was constructed at the completion of nine sessions.

Threshold sweep speed was calculated by fitting logistic curves as psychometric functions to the data with sweep speed being represented on a log scale. Curve fitting was performed using the LevenbergMarquardt algorithm (SigmaPlot curve-fitting software, SPSS, Chicago, IL) with slope and upper limit being free parameters in the nonlinear least-squares fit. Two different thresholds were determined from the psychometric functions: first, the threshold at $75 \%$ performance (hit rate 0.75 ) was determined. Second, the inflection point of the psychometric function was determined as a threshold, which is also the middle between top and bottom level of performance.

In the second part of the study we used a $2 \mathrm{AFC}$ paradigm with an adaptive method to obtain the animals' thresholds for different variations of the basic stimulus (varied in frequency content and sweep range). Adaptive methods vary the test parameter based on the observer's previous response. They are fast and efficient, as most parameter values are presented close to threshold. Only the threshold is determined directly by the procedure, not the whole psychometric function. We used a method ("Best PEST", best parameter estimation by sequential testing) that selects the next stimulus by applying a maximum likelihood approach (Lieberman and Pentland 1982; Hulse 1995). The test value for a trial is selected based on information gathered in all previous trials. Best PEST assumes a logistic distribution as the family of models for which maximum likelihood fits are sought and is more similar to standard QUEST than to traditional PEST techniques (Pokorny 1998). A prerequisite is a known general form of the psychometric function. The procedure was terminated when $90 \%$ confidence was reached for the current test value (interval included \pm 2 additional values) being the actual threshold. In most cases, however, animals reached $95 \%$ confidence.

Rats were trained for each new set of stimuli until they showed stable performance. Then, the threshold estimation was repeated twice for each stimulus configuration. We tested FM stimuli of three different sweep ranges and four different lower frequency boundaries. Note that changing lower frequency boundary for a given sweep range means changing center frequency and upper frequency boundaries in parallel. Lower frequency boundary is given as the relevant stimulus parameter, as this is most probably the limiting factor for perception in this case (see Discussion). Acquired values were compared in a three-factor ANOVA (two replicates) of a fractional factorial design with the factors of animal (four animals), lower frequency boundary of the FM (four values: 1024, 2048, 4096, and $8192 \mathrm{~Hz}$ ), and FM sweep range (three values: 2, 3, or 5 octaves). Some combinations in the analysis were missing as FM stimuli with a sweep range of 5 octaves were only tested at a lower frequency boundary of $1024 \mathrm{~Hz}$ (three combinations missing), and the 3-octave FM sweeps were not tested with a lower frequency boundary of $8192 \mathrm{~Hz}$ (one combination missing). Due to these missing combinations, the interaction between sweep range and lower frequency boundary could not be tested in the ANOVA. Statistical analysis was done using the statistics software JMP (Version 4.0; SAS Institute Inc., Cary, NC). Multiple comparisons of group means were performed using TukeyKramer honestly significant difference (HSD) test $(P<0.05)$.

\section{RESULTS}

The ability of rats to discriminate between upward and downward FM sweep direction depending on sweep speed was determined in five animals using a 2AFC paradigm with the method of constant stimuli. FM stimuli were presented from a loudspeaker above the animals. Psychometric functions were determined using wide-ranging logarithmic FM sweeps of five octaves $(1,024$ to $32,768 \mathrm{~Hz})$ with 13 different sweep speeds between 20 and 1,000 octaves/s (logarithmic spacing). The duration of the frequencymodulated part in the stimuli was between 250 and $5 \mathrm{~ms}$, respectively.

Well-trained animals that showed stable discrimination performance were tested in daily sessions that lasted up to $60 \mathrm{~min}$ (Fig. 1). Rats performed during these sessions on average around 700 trails (includ- 

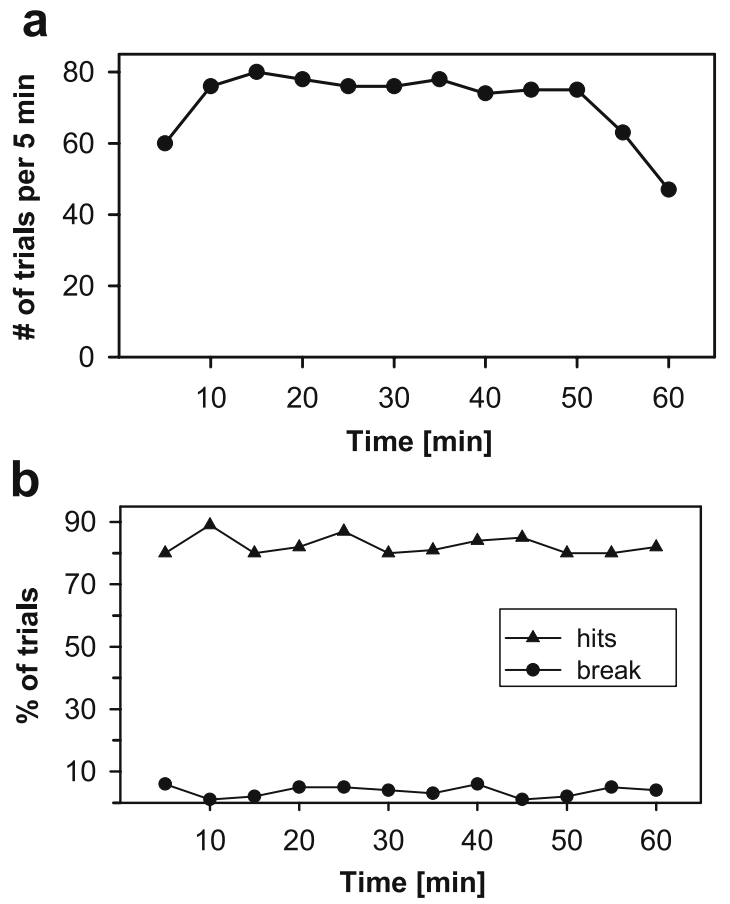

FIG. 1. Time course of performance of rat $L$ in one session (duration $60 \mathrm{~min}, 858$ trials) when discriminating between upward and downward FM tones of different sweep speed (20 to 1,000 octaves/s). The animal was well-trained in the discrimination paradigm (2AFC-procedure). Stimuli were presented with the method of constant stimuli. a Time course of trial frequency depicted as number of trials in 5-min blocks. The animal was constantly performing at a high level of trial frequency for about 50 min. b Time course of the percentage of hits and break errors during the session. Remember that hit rate level is determined by the mixture of easy and hard to discriminate FM stimuli (13 different sweep speeds).

ing hits, fault and break errors). They were allowed to initiate trials at their own speed, thereby performing usually at a constantly high level of trial frequency for about 50 to $60 \mathrm{~min}$. The rats' ability to discriminate stimuli was in most cases high even when trial frequency was low at the beginning or end of the session.

Individual psychometric functions showing the ability to discriminate between upward and downward FM sweep direction depending on sweep speed are shown in Figure 2. The five rats were tested until at least 200 discrimination decisions per sweep speed were acquired. This took up to nine sessions of testing. The slope part of the psychometric function was located in the range between 50 and 500 octaves/s for all animals. Individual differences are most obvious in the upper boundary of performance at low sweep speeds. Average hit rates at these easy to discriminate sweep speeds varied between 0.78 (rat N) and 0.94 (rat L), probably indicating different levels of non-perceptual influences such as general motivation between animals. The psychomet- ric function in one animal (rat P) had a clearly shallower slope than the others. An average psychometric function based on data from all five animals is shown in Figure 3. Note the logarithmic representation of
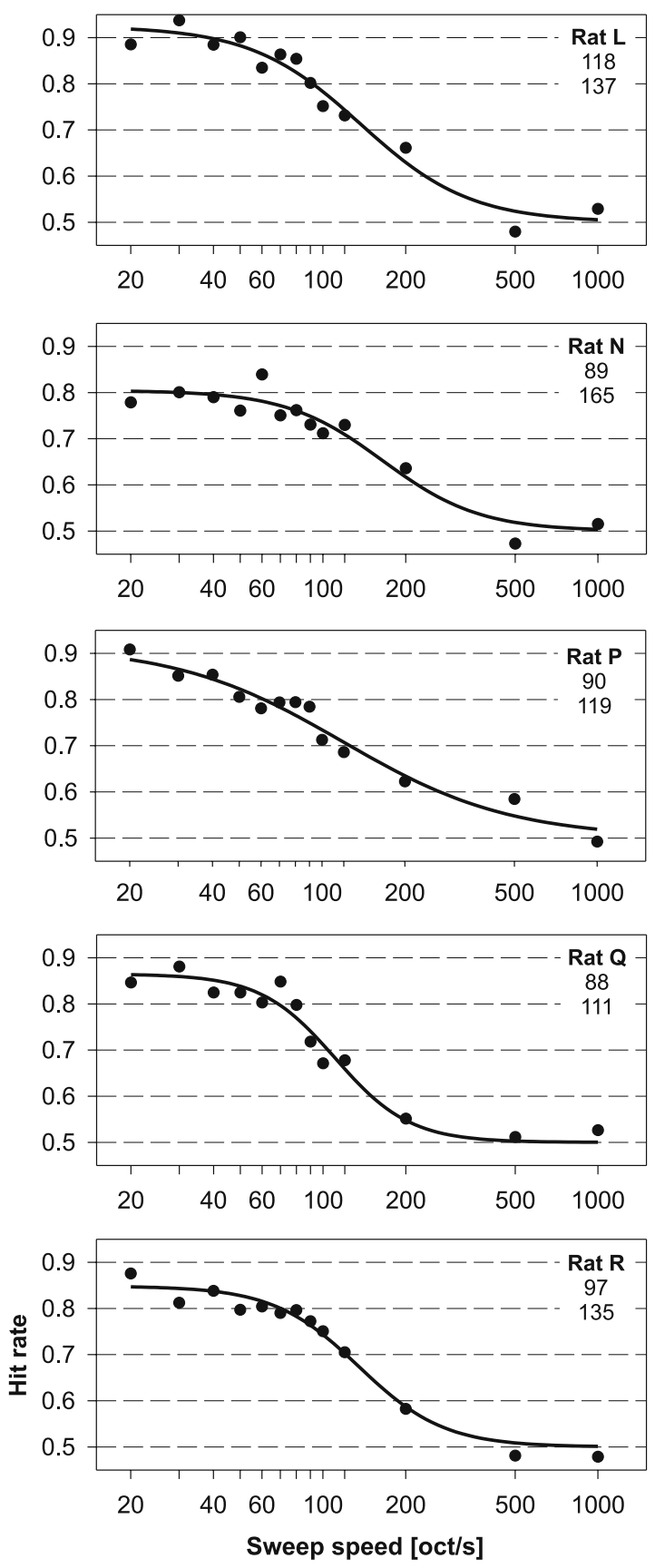

FIG. 2. Ability to discriminate FM sweep direction in logarithmic FM tones spanning five octaves by rats. Rates of correct responses (hit rate) are shown for sweep speeds between 20 and 1,000 octaves/s. Psychometric functions fitted to the data are depicted for five individual animals (rat L-rat R). Threshold sweep speed values (in octaves/s) for two different threshold measures as extracted from the psychometric functions are given in each panel below the animal name. Upper value: threshold at 0.75 hit rate, lower value: threshold sweep speed at the inflection point of the psychometric function (i.e., in the middle between minimum and maximum hit rates). 


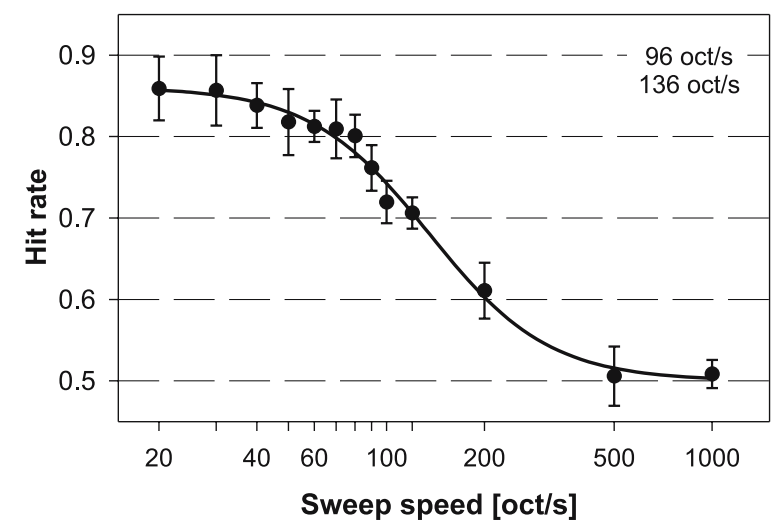

FIG. 3. Average ability of rats to discriminate between upward and downward FM sweep direction in logarithmic FM tones depending on sweep speed. The averaged psychometric function is based on data from five animals, individual variability is indicated by $95 \%$ confidence intervals. Two values for threshold sweep speed were determined from the data and are given in the panel: threshold at 0.75 hit rate (upper value) and the threshold at the inflection point of the average psychometric function (i.e., the middle between minimum and maximum hit rate; lower value).

FM sweep speed in auditory perception that leads to a symmetrical psychometric function and a good fit to the performance data on a log scale.

The 0.75 -threshold values for FM direction discrimination were between 88 and 118 octaves/s (Fig. 2), the threshold derived from the average psychometric function was 96 octaves/s (Fig. 3). These values are the ones that can be compared to the thresholds determined in the second part of this study using the Best PEST adaptive procedure. An important factor limiting the ability to discriminate fast FM tones is presumably the duration of the FM. The respective duration of the five-octave frequency modulation at this threshold was $52 \mathrm{~ms}$. An alternative threshold can be determined from the inflection point of the psychometric function (i.e., halfway between maximal performance and chance level), thereby taking into account the limiting influence of general, nonsensory factors such as attention or general motivation on the psychometric function. The average threshold determined this way was at a sweep speed of 136 octaves/s (respective length of FM sweep $37 \mathrm{~ms}$ ).

Influences of different stimulus parameters on the discrimination thresholds were investigated in a second set of experiments following the determination of basic psychometric functions. Determining the form of the psychometric function was an important prerequisite to test in a very efficient way the influence of additional parameters that limit discrimination performance. We used an adaptive procedure of threshold estimation in this part of the study (Best PEST) and collected data from four of the five animals that had participated in the first part.
One animal was not able to provide stable results for adaptive threshold estimation, especially when the discrimination became harder (small FM excursion, high frequency range). The procedure relies on a stable performance of 0.75 hit rate at a presumed threshold level for several trials. This seems to be a critical demand on animals that is hard to fulfill. However, after introducing a new set of stimuli for threshold estimation animals needed usually only few (2-3) training sessions until they had reached stable performance.

First, we compared thresholds determined from the psychometric functions (i.e., using the method of constant stimuli) to adaptively estimated thresholds from the Best PEST-procedure for stimuli of the same sweep range $(1,024-32,768 \mathrm{~Hz})$ with varying sweep speed (Fig. 4). Adaptively estimated thresholds were in all cases at higher sweep speeds (i.e., FM discrimination was better) than the ones determined from psychometric functions (mean 151.3 octaves/s compared to 96 octaves/s, respectively), although most animals needed only few training sessions until showing stable performance.

Stimuli of varying sweep range (two and three octaves in addition to the five-octave stimulus) and frequency content (lower frequency boundary 1,024; 2,048; 4,096 and 8,192 Hz) were tested after that in randomized order. Thresholds were determined for all possible stimulus types (i.e combinations of sweep range and lower frequency boundary) with an upper frequency boundary of up to $32,768 \mathrm{~Hz}$ (two octaves above 1,024; 2,048; 4,096; and $8192 \mathrm{~Hz}$; three octaves above 1,$024 ; 2,048$ and 4,096 Hz). For each stimulus type, we first trained the animals until showing stable performance. Then, two thresholds per stimulus configuration were determined in two sessions.

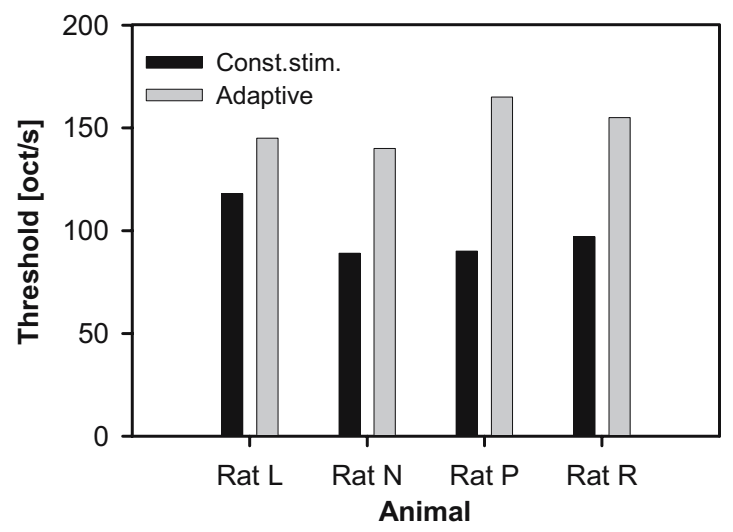

FIG. 4. Comparison of threshold sweep speeds of thresholds determined from psychometric functions (black bars) to thresholds measured adaptively with the Best PEST-method (gray bars) for four animals. Logarithmic FM tones with a five-octave frequency range $(1,024 \mathrm{~Hz}$ to $32,768 \mathrm{~Hz})$ were used with varying sweep speed. 
Significant effects of the parameters "animal" (influence of individual animals), "lower frequency boundary" and "sweep range" on threshold sweep speed of FM stimuli were found in a 3-factor ANOVA (ANOVA Type III, SS; Whole model: $F_{8,55}=81.6, R^{2}=$ $92.2 \%)$. In spite of a clear effect of the parameter animal $\left(F_{3,55}=46.8, P<0.0001\right)$, we found no significant interaction of this parameter with the stimulus-related parameters (lower frequency boundary, sweep range) tested $(P>0.31$, interaction-term removed for further analysis).

Significant influences on threshold sweep speed were also found in this ANOVA for the FM stimulus parameters sweep range $\left(F_{3,55}=58.0, P<\right.$ 0.0001 , Fig. 5$)$ and lower frequency boundary $\left(F_{3,55}=\right.$ 78.7, $P<0.0001$, Fig. 6). Discrimination improved (i.e., threshold sweep speeds were significantly increased) for stimuli with larger sweep range, as revealed by multiple comparisons (Fig. 5). One has to keep in mind that extended sweep range is accompanied by a strong increase in the temporal duration of the FM stimulus. The influence of the lower frequency boundary of FM stimuli (Fig. 6) was characterized by a significant deterioration in discrimination (i.e., decrease in threshold sweep speed) when the lower frequency boundary was raised from 2 to $4 \mathrm{kHz}$ or higher $(P<0.05$, Tukey-Kramer HSD test). This indicated an advantage of FM stimuli with frequency content in the low frequency range for FM discrimination performance.

The influence of the detailed structure of the FM stimuli on threshold estimation was investigated in a final experiment. FM stimuli used in both parts of

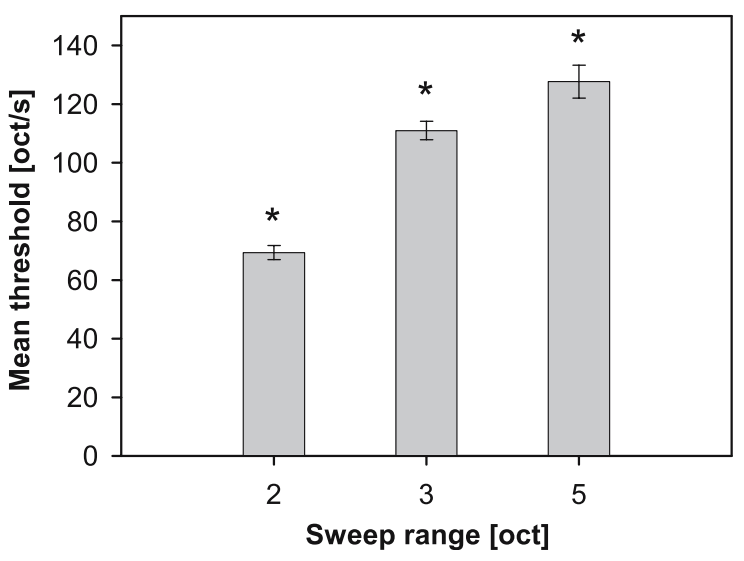

FIG. 5. Influence of sweep range on discrimination of FM sweep direction depending on sweep speed. Threshold sweep speeds (in octaves/s) are given as the least square means ( \pm standard error) from an ANOVA analysis for two-, three-, or five-octave FM stimuli (see text). Stars indicate significant differences of each mean value against all others as revealed by multiple comparisons $(P<0.05$, Tukey-Kramer HSD test).

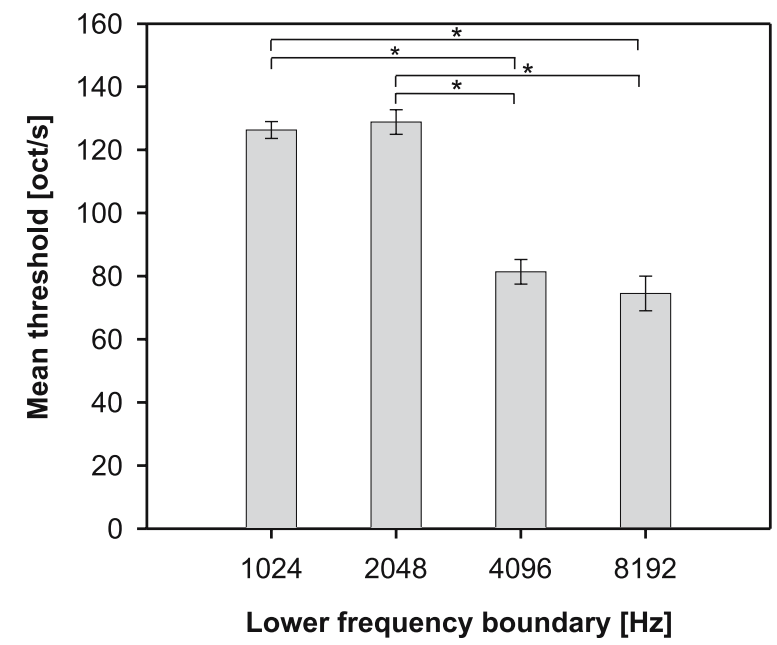

FIG. 6. Influence of the lower frequency boundary of FM stimuli on discrimination performance. Threshold sweep speeds (in octaves/s) are given as the least square means ( \pm standard error) from an ANOVA analysis (see text). Multiple comparisons $(P<$ 0.05, indicated above bars) revealed significantly reduced mean thresholds for stimuli with high frequency content (lower frequency boundary $4 \mathrm{kHz}$ or higher) compared to stimuli with additional low frequency content (lower frequency boundary $2 \mathrm{kHz}$ or lower).

this study had a central frequency-modulated part (sweep) with an appended constant frequency (CF) section during stimulus rise and fall time $(5 \mathrm{~ms})$. This was done in order to prevent any interaction between the (in some cases rather short) frequency modulation and stimulus on- and offset. The CF-part, on the other hand, might have been used by the animals to solve the discrimination task by simply comparing the start frequency with the stop frequency in the stimulus. This possibility was tested by determining in Best PEST-procedures the threshold sweep speed for discrimination performance of FM stimuli with CF-part and of FM stimuli without CF-part. Fiveoctave FM sweeps (1,024-32,767 Hz) were used in both cases. The usual range of sweep speeds was presented in the adaptive procedure (see Methods). Mean threshold sweep speeds for FM discrimination were comparable to the data shown above. With the thresholds for FM stimuli without CF-part being on average only 1.25 octaves/s higher, discrimination performance was not significantly different between these two types of stimuli (Paired $t$-test, n.s.).

\section{DISCUSSION}

Investigating the psychoacoustics of FM discrimination in an animal model, the rat, was mainly motivated by the need for data at the perceptual level that can be related to the well investigated underlying physiological mechanisms of neuronal FM encoding. 
The perception of sweep directions in FM stimuli probably depends on neurons that specifically encode the directions and/or sweep speeds of FM stimuli in the mammalian brain. Such neurons at the level of the auditory cortex are especially important. This is indicated by the fact that FM discrimination depends on their functional integrity (Kelly and Whitfield 1971; Wetzel et al. 1998a). In addition, an auditory cortical structure that specifically processes FM stimuli or 'spectral motion' was described in humans (Thivard et al. 2000; Behne et al. 2005).

In the rat, large proportions of FM encoding neurons were found in auditory cortex (Gaese and Ostwald 1995; Ricketts et al. 1998; Zhang et al. 2003) and inferior colliculus (Felsheim and Ostwald 1996). Specific response properties of these neurons correspond well to properties of discrimination performance at the behavioral level, as they are described in this study. Several neuronal characteristics can be related to the deteriorating sweep speed-dependent FM discrimination performance between 60 and 300 octaves/s (see Fig. 3): first, the distribution of preferred FM sweep speeds in neurons at the cortical level has a maximum at 70 octaves/s and an upper steep slope between 100 and 500 octaves/s (Zhang et al. 2003). Second, the upper slope of the distribution of preferred FM sweep speeds at the level of the inferior colliculus has a maximum at around 65 octaves/s and an upper 90\%-limit at around 400 octaves/s (Felsheim and Ostwald 1996). Finally, the upper limit of neuronal selectivity for sweep direction matches the upper limit for FM direction discrimination in rats, which might be even more relevant for this perceptual task: directional selectivity is strong around 70 octaves/s in rat auditory cortical neurons and is limited to sweep speeds below 200 to 500 octaves/s depending on the measure used (Zhang et al. 2003).

Additional factors that limit FM directional discrimination in rats were described in the second part of this study. An influence of FM sweep range (varied between two and five octaves) was shown, with increasing FM sweep range facilitating directional discrimination. Both factors, sweep speed and sweep range are directly related to the temporal duration of the FM sweep. This is most probably the one FM parameter that is limiting discrimination in fast FM sweeps. Duration can either be increased by increasing FM sweep range or by decreasing sweep speed. Both types of increase in duration lead to an improvement in FM discrimination, as has already been shown in humans (Cullen and Collins 1982; Schouten 1985).

FM directional discrimination also improved with decreasing lower frequency boundary of FM sweeps, with an especially strong improvement, when the lower frequency boundary of FM stimuli was lowered from 4 to $2 \mathrm{kHz}$. Note, that exactly the same pattern would have been produced related to center frequency or upper frequency boundary of the FM sweep, only shifted towards slightly higher frequency values. Several lines of evidence can possibly explain the pattern. A first possibility would be the influence of auditory threshold. While the pattern found cannot be simply related to general hearing sensitivity, as the rat is up to $15 \mathrm{~dB}$ more sensitive in the range between 4 and $32 \mathrm{kHz}$ compared to the range below $4 \mathrm{kHz}$ (Kelly and Masterton 1977), frequencydependent changes in audibility might have an effect. The steep auditory threshold between 1 and $8 \mathrm{kHz}$ (around $10 \mathrm{~dB} /$ octave) might induce a gradient in loudness along the frequency axis that would enhance FM directional discrimination. The strong drop in FM discrimination performance between 2 and $4 \mathrm{kHz}$ found in this study could then be related to the fact that only FM stimuli with 1 or 2 $\mathrm{kHz}$ lower frequency boundary swept to greater extend through the frequency range below $8 \mathrm{kHz}$, whereas stimuli with $4 \mathrm{kHz}-$ lower frequency boundary did not. As a second possibility, the effects might be caused by differences in selectivity of auditory neurons along the frequency axis. Among them are frequency-dependent differences in the width of frequency response areas in the periphery (el Barbary 1991). Rather broad frequency response areas of lowfrequency neurons could explain improved FM discrimination in the low-frequency range only. Additional characteristics might be caused by frequency-specific interactions of excitation and inhibition at the cortical level (Zhang et al. 2003).

Any of the frequency-dependent mechanisms for FM discrimination described so far should not only be obvious at the behavioral level, but also in the encoding of FM stimuli at higher levels of the central auditory pathway. The pattern there can be seen as the combined result of all different kinds of frequencydependent effects along the ascending auditory pathway. Fortunately, good data on encoding of FM sweeps in the rat auditory cortex are provided by a recent study (Zhang et al. 2003). They found direction selectivity to be weak in the intermediate frequency range between 8 and $16 \mathrm{kHz}$, but good with a preference for upward FM below that range and also good with preference for downward FM at higher frequencies. Thus, the pattern of FM encoding along the frequency axis at the cortical level can explain the deteriorated direction discrimination in the intermediate frequency range. It would, however, predict good discrimination also above $8 \mathrm{kHz}$, which we did not find. Moreover, other electrophysiological studies found no indication of such a frequency-specific 
reduction in neuronal direction selectivity in the rat (Felsheim and Ostwald 1996; Ricketts et al. 1998).

The deteriorated discrimination performance at frequencies above $4 \mathrm{kHz}$ might, as a third explanation, suggest that FM directional discrimination relies on a temporal code derived from phase-locking, which is only available below $4 \mathrm{kHz}$. The importance of this mechanism in addition to a place code has been a matter of debate in human psychophysics for a long time. Most of the studies using slow FM sweeps (see Introduction) found either no evidence for a temporal mechanism (Madden and Fire 1997; Moore and Sek 1998) or just small indications (Sek and Moore 2000). Investigations using fast FM sweep speeds, comparable to this study, tested only in the frequency range below $3 \mathrm{kHz}$. In summary, any of the possible mechanisms presented here might add to the perceptual advantage in the low frequency range. Determining the underlying mechanisms for fast FM discrimination in general is necessary before the limiting factors can be specified in more clearly.

Fast frequency modulations (FM) are major components of behaviorally relevant signals, such as species-specific vocalizations. In rats, especially one type of vocalization (" $50-\mathrm{kHz}$ vocalizations") has prominent frequency-modulated parts. These vocalizations consist of individual frequency-modulated calls up to $65 \mathrm{~ms}$ duration in the frequency range between 35 and $70 \mathrm{kHz}$ (Kaltwasser 1990). Behaviorally, these vocalizations are related to playful behavior in juvenile rats (Knutson et al. 1998), they can be driven by social contact, and were related to behavioral states of reward anticipation (Brudzynski and Pniak 2002). Transient components include upward and downward FM components with sweep speeds up to $40-50$ octaves/s. This is well in the range of good directional discrimination performance, as it was determined in this study. While in rats it can only be assumed that these FM components are bearing important information, this has been investigated in detail in other species. One of the most intriguing example comes from auditory mate recognition in penguins (Aubin 2004). King penguins (Aptenodytes patagonicus) returning to the colony find their mate among thousands of vocalizing conspecifics using a display call with individually distinctive features. Recognition is mainly based on the FM profile (direction, slope) of the call, in particular its initial inflection (Lengagne and Aubin 2000).

The methods of training and threshold determination applied in this study were in some ways not following the usual approach of psychoacoustical experiments in rodents. Regarding the training procedure, we made use of a positive reinforcement schedule (reward) for psychophysical threshold esti- mation. This had previously been used only in few cases in the rat (e.g., Talwar and Gerstein 1998) as compared to the traditionally used fear-conditioning procedures of negative reinforcement (Kelly and Masterton 1977; Heffner and Heffner 1985). As an advantage, positive reinforcement procedures, especially if they are combined with self-initiated trials by the animal, can lead to rather high trial numbers per daily session (500 to 1500 trials). Perceptual testing on vision in primates commonly takes advantage of this (e.g., Mandon and Kreiter 2005). Large trial numbers are a necessary prerequisite when trying to directly relate perceptual performance to its underlying neuronal functions in combined experiments (Salzman and Newsome 1994).

As a possible disadvantage, however, positive reinforcement might be seen as less demanding compared to fear-conditioning. This might have been the cause for the less than optimal hit rates at low FM sweep speeds in some animals (e.g., Rat N in Figure 2). Such upper levels of performance being clearly below $100 \%$ can be found sometimes in animal investigations (Schmidt 1995). Only rat N showed a small response bias at slow sweep speeds, that was a partial cause of that. The main cause was some nonperceptual effect, presumably related to the general level of motivation. As a result, thresholds might have been slightly underestimated. Because of that, thresholds at the inflection point of the psychometric function (i.e., in the middle between minimum and maximum hit rate) are given for comparison. They take into account the reduced maximal performance of the animals (Schmidt 1995) and are indeed slightly higher. These thresholds should be well comparable to thresholds determined on a negative reinforcement schedule. Interestingly, threshold sweep speeds determined in the adaptive procedure that tracks a hit rate of 0.75 , were even higher than the two types of threshold estimates from psychometric functions (range 140-165, mean 151.3 octaves/s). The described influences of sweep range and lower frequency boundary on discrimination performance are not confound by the possible underestimation. They are based on relative changes in threshold sweep speed and were measured using the adaptive procedure only, i.e., under constant conditions and at a very short time-scale.

Besides the method of training also the procedure for stimulus presentation that we applied was unusu$\mathrm{al}$, as psychoacoustical investigations in animals do not use very often 2AFC procedures. The 2AFC procedures have the advantages of providing a criterion-free measure of detectability (Penner 1995) and an unambiguous response categorization (Hulse 1995), but confront the animal with a much 
harder task to solve than the usually used Go/NoGo task. In a Go/NoGo task in each trial a train of stimuli is presented, that includes one (to detect) deviant. This allows for a direct comparison of neighboring stimuli inside the train, which is easy and might just be based on a simple concept of "different" without generalization. In 2AFC procedures in animals, on the other hand, just one stimulus is presented per trial (either upward or downward FM sweep). In each trial, the rats had to compare this stimulus to a general concept of "upward FM" vs. "downward FM" stored in memory for correct discrimination. Thus, it was indeed the performance of FM directional discrimination that was determined in this study.

In view of the advantages noted above, the question remains if 2AFC procedures are efficient enough for a broad application. An important drawback of forced choice experiments, when the number of alternatives is limited to two, is the fact, that the necessary number of trials to reach a certain precision must be increased at least by a factor of 2-3 compared to a yes-no (i.e., Go/NoGo) design (Treutwein 1995). After having shown the feasibility of a 2AFC-procedure in combination with the method of constant stimuli, we reduced the necessary number of trials strongly by applying an adaptive procedure for threshold estimation when we tested the influence of various stimulus parameters. Animals still needed comparably large numbers of trials for threshold estimation in the adaptive Best PEST procedure (on average 313.7 trials). This was due to the rather conservative statistical criterion for an indication of a true threshold estimation (confidence level of 0.95) that was included in the procedure. However, as the rats were performing quite fast in the tests (about 500 to 700 trials in $1 \mathrm{~h}$ ), it was still possible to determine a threshold once or (sometimes) twice in a daily session. Based on the experience gained in these experiments we can further optimize the procedure for threshold estimation by, for example, limiting the number of trials as it is sometimes done when human patients are tested. Combining the different approaches leads, in summary, to a method for precise and efficient threshold estimation (Phipps et al. 2001).

\section{ACKNOWLEDGMENTS}

The authors acknowledge Dr. Vazha Amiranashvilli for very skillful computer programming of a computer system for behavioral testing and sound delivery and Dr. Katrin Böhning-Gaese for statistical advice. We also thank Anna Wittekindt for reading previous versions of the manuscript. Financial support was provided by the DFG (SPP 1001, "Sensomotorische Integration").

\section{REFERENCES}

Aubin T. Penguins and their noisy world. An. Acad. Bras. Cienc. 76:279-283, 2004.

Behne N, Scheich H, Brechmann A. Contralateral white noise selectively changes right human auditory cortex activity caused by a FM-direction task. J. Neurophysiol. 93:414-423, 2005.

Brudzynski SM, PNiAK A. Social contacts and production of $50-\mathrm{kHz}$ short ultrasonic calls in adult rats. J. Comp. Psychol. 116:73-82, 2002.

Cullen JK, Collins MJ. Audibility of short-duration tone-glides as a function of rate of frequency change. Hear. Res. 7:115-125, 1982.

Dooley GJ, Moore BCJ. Detection of linear glides as a function of frequency and duration. J. Acoust. Soc. Am. 84:2045-2057, 1988.

EL BARBARY A. Auditory nerve of the normal and jaundiced rat. 2. Frequency selectivity and two-tone rate suppression. Hear. Res. 54:91-104, 1991.

Felsheim C, Ostwald J. Responses to exponential frequency modulations in the rat inferior colliculus. Hear. Res. 98:137151, 1996

Friauf E. Tonotopic order in the adult and developing auditory system of the rat as shown by c-fos immunocytochemistry. Eur. J. Neurosci. 4:798-812, 1992.

Gaese BH, Ostwald J. Temporal coding of amplitude and frequency modulation in the rat auditory cortex. Eur. J. Neurosci. 7:438-450, 1995.

Gaese BH, Ostwald J. Complexity and temporal dynamics of frequency encoding in the awake rat auditory cortex. Eur. J. Neurosci. 18:2638-2652, 2003.

GARDNER RB, WiLson JP. Evidence for direction-specific channels in the processing of frequency modulation. J. Acoust. Soc. Am. 66:704-709, 1979.

Gellermann LW. Chance orders of altering stimuli in visual discrimination experiments. J. Gen. Psychol. 42:206-208, 1933.

HAge SR, Ehret G. Mapping responses to frequency sweeps and tones in the inferior colliculus of house mice. Eur. J. Neurosci. 18:2301-2312, 2003.

Heffner HE, Heffner RS. Sound localization in wild Norway rats (Rattus norvegicus). Hear. Res. 19:151-156, 1985.

Heil P, Langner G, Scheich H. Processing of frequency-modulated stimuli in the chick auditory cortex analogue: evidence for topographic representations and possible mechanisms of rate and directional sensitivity. J. Comp. Physiol., A 171:583-600, 1992a.

Heil P, Rajan R, Irvine DRF. Sensitivity of neurons in cat primary auditory cortex to tones and frequency-modulated stimuli. I: effects of variation of stimulus parameters. Hear. Res. 63:108134, 1992b.

HulsE SH. The discrimination-transfer procedure for studying auditory perception and perceptual invariance in animals. In: Klump GM, Dooling RJ, Fay RR, Stebbins WC (eds) Methods in Comparative Psychoacoustics. Birkhaeuser, Basel, pp 319-330, 1995.

Kaltwasser MT. Acoustic signaling in the black rat (Rattus rattus). J. Comp. Psychol. 104:227-232, 1990.

Kelly JB, Masterton B. Auditory sensitivity of the albino rat. J. Comp. Physiol. Psychol. 91:930-936, 1977.

Kelly JB, Whitfield IC. Effects of auditory cortical lesions on discrimination of rising and falling frequency-modulated tones. J. Neurophysiol. 34:802-816, 1971.

Knutson B, Burgdorf J, PANksepe J. Anticipation of play elicits highfrequency ultrasonic vocalizations in young rats. J. Comp. Psychol. 112:65-73, 1998. 
Lengagne T, Aubin T. Perceptual salience of individually distinctive features in the calls of adult king penguins. J. Acoust. Soc. Am. 107:508-516, 2000.

Liberman AM, Mattingly IG. A specialization for speech perception. Science 243:489-494, 1989.

Lieberman HR, Pentland AP. Microcomputer-based estimation of psychophysical thresholds: the best PEST. Behav. Res. Meth. Instrum. 14:21-25, 1982.

Madden JP, Fire KM. Detection and discrimination of frequency glides as a function of direction, frequency span and center frequency. J. Acoust. Soc. Am. 102:2920-2924, 1997.

Mandon S, KreIter AK. Rapid contour integration in macaque monkeys. Vis. Res. 45:291-300, 2005.

MARLER P. Bird calls: their potential for behavioral neurobiology. Ann. N. Y. Acad. Sci. 1016:31-44, 2004.

May B, Moody DB, Stebbins WC. Categorial perception of conspecific communication sounds by Japanese macaques, Macaca fuscata. J. Acoust. Soc. Am. 85:837-847, 1989.

Mendelson JR, Cynader MS. Sensitivity of cat primary auditory cortex (A1) neurons to the direction and rate of frequency modulation. Brain Res. 327:331-335, 1985.

Mendelson JR, Schreiner CE, Sutter ML, Grasse KL. Functional topography of cat primary auditory cortex: responses to frequency-modulated sweeps. Exp. Brain Res. 94:65-87, 1993.

Mercado E, Orduna I, Nowak JM. Auditory categorization of complex sounds by rats (Rattus norvegicus). J. Comp. Psychol. 119:90-98, 2005.

MøLLER AR. Unit responses in the cochlear nucleus of the rat to sweep tones. Acta Physiol. Scand. 76:503-512, 1969.

Moody DB, May B, Cole DM, Stebbins WC. The role of frequency modulation in the perception of complex stimuli by primates. Exp. Biol. 45:219-232, 1986.

Moore BCJ, Seк A. Discrimination of frequency glides with superimposed random glides in level. J. Acoust. Soc. Am. 104:411421, 1998.

MÜLLER M. Frequency representation in the rat cochlea. Hear. Res. 51:247-254, 1991.

Nelken I, Versnel H. Responses to linear and logarithmic frequency-modulated sweeps in ferret primary auditory cortex. Eur. J. Neurosci. 12:549-562, 2000.

Penner MJ. Psychophysical methods. In: Klump GM, Dooling RJ, Fay RR, Stebbins WC (eds) Methods in Comparative Psychoacoustics. Birkhäuser, Basel/Switzerland, pp. 47-57, 1995.
Phipps JA, Zele AJ, Dang T, Vingrys AJ. Fast psychophysical procedures for clinical testing. Clin. Exp. Optom. 84:264-269, 2001.

Pokorny RA. Using maximum-likelihood adaptive methods to estimate difference thresholds and point of subjective equality. Behav. Res. Meth. Instrum. 30:624-636, 1998.

Ricketts C, Mendelson JR, Anand B, English R. Responses to timevarying stimuli in rat auditory cortex. Hear. Res. 123:27-30, 1998.

Sales GD, Pye JD. Ultrasonic Communication by Animals. Chapman and Hall, London, 1974.

Salzman CD, Newsome WT. Neural mechanisms for forming a perceptual decision. Science 264:231-237, 1994.

Schmidt S. Psychoacoustic studies in bats. In: Klump GM, Dooling RJ, Fay RR, Stebbins WC (eds) Methods in Comparative Psychoacoustics. Birkhäuser, Basel, pp. 123-134, 1995.

SChNitzler HU, Kalko EKV. Echolocation by insect-eating bats. Bioscience 51:557-569, 2001.

Schouten MEH. Identification and discrimination in sweep tones. Percept. Psychophys. 37:369-376, 1985.

Sek A, Moore BCJ. Detection of quasitrapezoidal frequency and amplitude modulation. J. Acoust. Soc. Am. 107:1598-1604, 2000.

Talwar SK, Gerstein GL. Auditory frequency discrimination in the white rat. Hear. Res. 126:135-150, 1998.

Thivard L, Belin P, Zilbovicius M, Poline JB, Samson Y. A cortical region sensitive to auditory spectral motion. Neuroreport 11:2969-2972, 2000.

Treutwein B. Adaptive psychophysical procedures. Vis. Res. 35:2503-2522, 1995.

Wetzel W, Ohl FW, Wagner T, Scheich H. Right auditory cortex lesion in Mongolian gerbils impairs discrimination of rising and falling frequency-modulated tones. Neurosci. Lett. 252:115118, 1998a.

Wetzel W, Wagner T, Ohl FW, Scheich H. Categorical discrimination of direction in frequency-modulated tones by Mongolian gerbils. Behav. Brain Res. 91:29-39, 1998b.

Whitfield IC, Evans EF. Responses of auditory cortical neurons to stimuli of changing frequency. J. Neurophysiol. 28:655-672, 1965.

Zhang LI, Tan AY, Schreiner CE, Merzenich MM. Topography and synaptic shaping of direction selectivity in primary auditory cortex. Nature 424:201-205, 2003. 